\title{
Endogenous plasticity in neuro-rehabilitation following partial spinal cord lesions
}

\author{
Bror Alstermark $^{1 *}$ and Lars-Gunnar Pettersson ${ }^{2}$ \\ 1 Section of Physiology, Department of Integrative Medical Biology, Umeå University, Umeå, Sweden \\ 2 Department of Physiology, Institute of Neuroscience and Physiology, Sahlgrenska Academy at University of Gothenburg, Gothenburg, Sweden
}

\author{
Edited by: \\ Yukio Nishimura, National Institute \\ for Physiological Sciences, Japan \\ Reviewed by: \\ Jennie Leach, University of \\ Maryland, USA \\ Brian R. Noga, University of Miami, \\ USA

\section{${ }^{*}$ Correspondence:} \\ Bror Alstermark, Section of \\ Physiology, Department of \\ Integrative Medical Biology, Umeå \\ University, S-901 87 Umeå, Sweden \\ e-mail: bror.alstermark@umu.se
}

Currently, much interest in neuro-rehabilitation is focused on mechanisms related to axonal outgrowth and formation of new circuits although still little is known about the functionality in motor behavior. This is a highly exciting avenue of research and most important to consider when dealing with large lesions. Here, we address endogenous mechanisms with the potential of modifying the function of already existing spinal circuits via associative plasticity. We forward a hypothesis based on experimental findings suggesting that potentiation of synaptic transmission in un-injured pathways can be monitored and adjusted by a Cerebellar loop involving the Reticulospinal, Rubrospinal and Corticospinal tracts and spinal interneurons with projection to motoneurons. This mechanism could be of relevance when lesions are less extensive and the integrity of the neural circuits remains in part. Endogenous plasticity in the spinal cord could be of clinical importance if stimulated in an adequate manner, e.g., by using optimal training protocols.

Keywords: rehabilitation, partial spinal cord lesion, grasping, corticospinal, rubrospinal, reticulospinal, interneuron, lateral reticular nucleus

\section{INTRODUCTION}

A major problem following injuries in the CNS is poor recovery of sensorimotor control leaving the subject substantially handicapped for the remaining life. However, there are several remarkable cases of recovery in the literature giving hope (Bach-y-Rita, 1981), but little or no explanation of the underlying mechanisms. Injuries to the spinal cord are often partial and can spare both long and short descending and ascending pathways. Based on experimental findings, we propose a hypothesis for potentiation of transmission in un-injured pathways and which involves spinal interneuronal networks, descending brainstem systems and the Cerebellum.

The spinal cord possesses microcircuits not only for control of reflexes, posture, respiration, locomotion, and scratching, but also for control of voluntary movements like reaching and grasping (cf. reviews and references therein; Baldissera et al., 1981; Alstermark and Lundberg, 1992; Alstermark and Isa, 2012). Behavioral experiments in the cat and monkey showed that reaching and grasping can be controlled via interneuronal circuits in the cervical spinal cord (Alstermark et al., 1981b, 2011; Sasaki et al., 2004). In those experiments, selective spinal cord lesions were made to delineate the different spinal interneuronal systems mediating the command for these movements to forelimb motoneurones and to investigate the control from different descending pathways. It was found that the Corticospinal (CST) and Rubrospinal (RuST) tracts played a major role in the control of reaching and grasping, to the extent that transection of them resulted in complete loss of these movements, whereas the Reticulospinal tract (ReST) did not play any significant role. However, in case of incomplete CST and RuST lesions, there was a fast and significant recovery that could be mediated via the ReST (Alstermark et al., 1987; cf. also Pettersson et al., 2007).

\section{BACKGROUND}

Figure 1A illustrates schematically the organization of two spinal interneuronal systems that can mediate the motor commands for reaching, C3-C4 propriospinal neurons ( $\mathrm{PN}$; labeled in blue) and grasping, C6-Th1 segmental interneurons (sINs; labeled in orange). Both systems can be controlled from the cortico- (CST; in black), rubro- (RuST; in red), and reticulospinal (ReST, in green) tracts. The RuST and ReST are controlled from the motor cortex as well as from the deep cerebellar nuclei (not shown). The PNs, sINs, and ReST project directly to the motoneurons (MN). In addition to their motoneuronal projections, the PNs and the sINs project to neurons in the precerebellar Lateral Reticular Nucleus (LRN; not shown). This is illustrated for the sINs in the summarizing Figure 4C.

The preoperative grasping performance is shown for the cat in Figure 1B when retrieving a morsel of food from a tube. Note the combined digit flexion, protrusion of the claws and supination of the wrist after withdrawal from the tube.

Figure 2A schematically shows a complete CST and RuST lesion made in the dorsal part of the lateral funiculus (DLF) in C5. Following this lesion, the cat completely lost the digit grasping movements as shown in Figure 2C obtained 7 days postoperatively. The diagram in Figure 2D (blue plots) illustrates the percentage of successful trials in different experiments during the first two postoperative weeks in four different cats with complete CST and RuST lesions. A slow recovery is evident, but the success rate remains lower than $50 \%$. In a similar test, but using less frequent training and allowing taking by the mouth of 


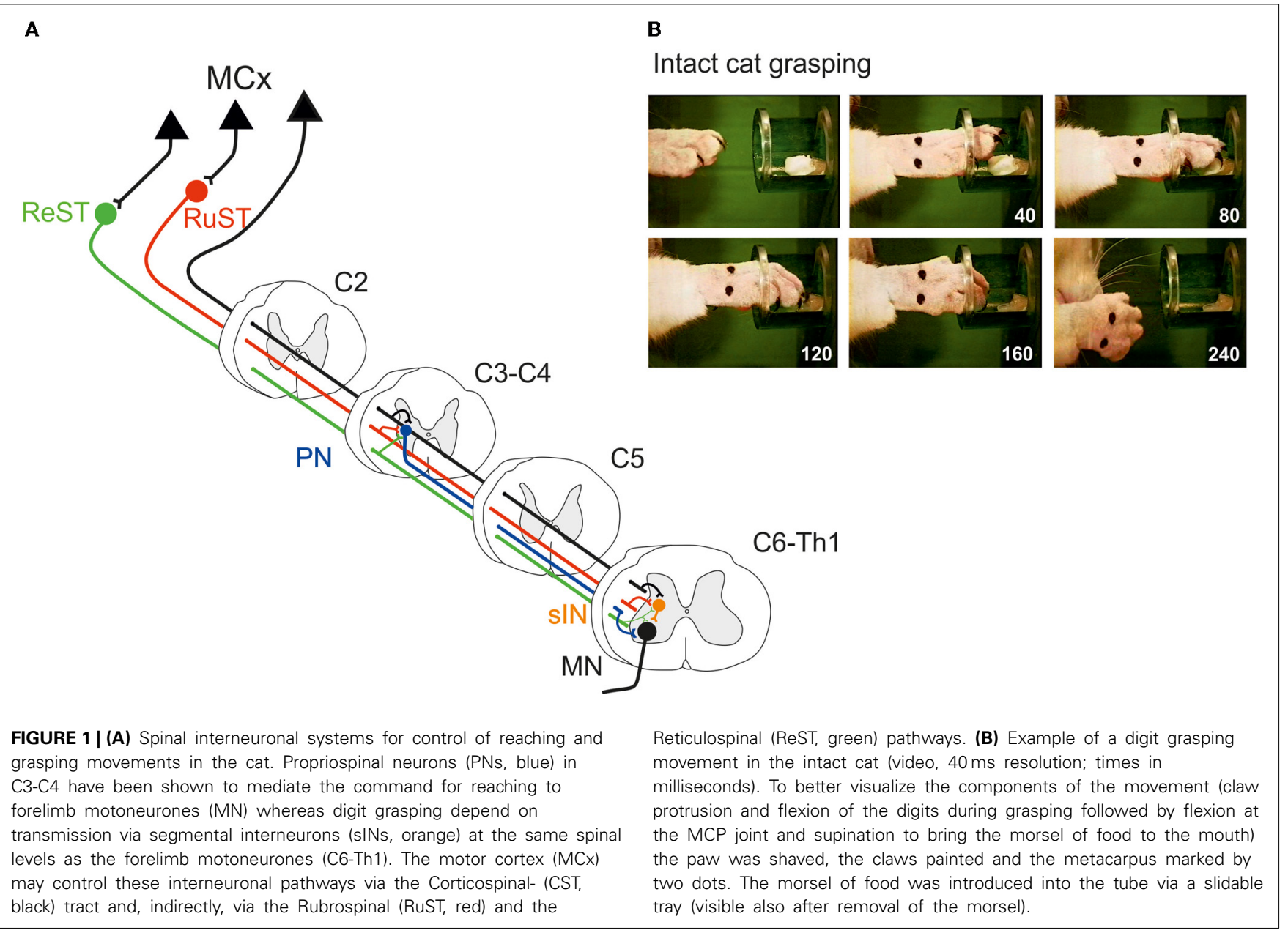

morsels dropped onto the floor in trials with insufficient grasping, Alstermark et al. (1981b) described an even slower recovery. Only weak toe flexion was observed, beginning from 2-4 weeks and the cats only reached a success rate of about $50 \%$ after 6 months. No successful trials were observed within the first 2 weeks (indicated by a black dot in Figure 2D). Presumably, the intensity, time of onset and behavioral paradigm are important for the time course of recovery as emphasized by Sugiyama et al. (2013).

In contrast, if some rubrospinal fibers escaped the lesion as shown in Figure 2B, the recovery was much faster and more complete. Alstermark et al. (1987) showed that if about 50\% of the RuST remained intact, as assessed by the amplitude of the descending volley recorded from dissected spinal halves caudal to the lesion, successful digit grasping was observed already in the first experiment 6 days postoperatively. Pettersson et al. (2000) showed that as little as $4 \%$ of the RuST was sufficient to markedly improve the recovery. The success rates for three cats with 4-6\% of remaining RuST are shown in Figure 2D (red plots). Already within the first week the success rate increased to about $70 \%$ of the preoperative value. Performance of digit grasping on the 7 th postoperative day is exemplified in Figure 2E. The findings are also summarized in the Supplementary Movie 1.
Alstermark et al. (1987) showed further that 1 month after the incomplete lesion, the remaining RuST fibers were no longer required for performance of the recovered digit grasping movement. If a second lesion of the DLF (denoted lesion II in Figure 3A) was added and which extended more ventrally to transect the remaining intact RuST fibers, digit grasping was present in the first trial 6 days postoperatively. Figure 3A schematically shows that experimental paradigm with two serial lesions of the DLF (lesion I and lesion II) and Figure 3B shows successful retrieval of the morsel of food 6 days after lesion II.

Finally, Alstermark et al. (1987) found that the recovered grasp function could be permanently abolished by adding a third lesion ventrally in C2 transecting the ReST. It was concluded that the intact ReST had taken over the role of the CST and RuST pathways. The RuST fibers remaining after lesion I were suggested to serve as a teacher facilitating the ability to use the ReST to command the movement.

How can such a functional takeover be achieved?

\section{HYPOTHESIS FOR FUNCTIONAL TAKEOVER FOLLOWING PARTIAL SPINAL CORD LESION}

It is a remarkable finding that as little as $4 \%$ remaining RuST fibers, after a DLF lesion, suffice for an improved functional 


\section{A Complete CST and RuST}

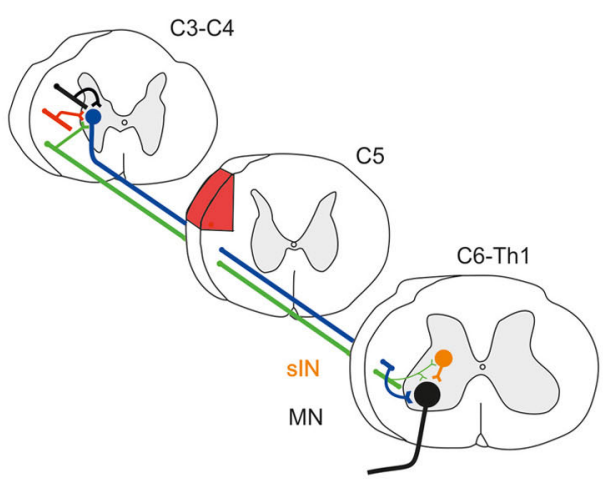

B Complete CST Partial RuST

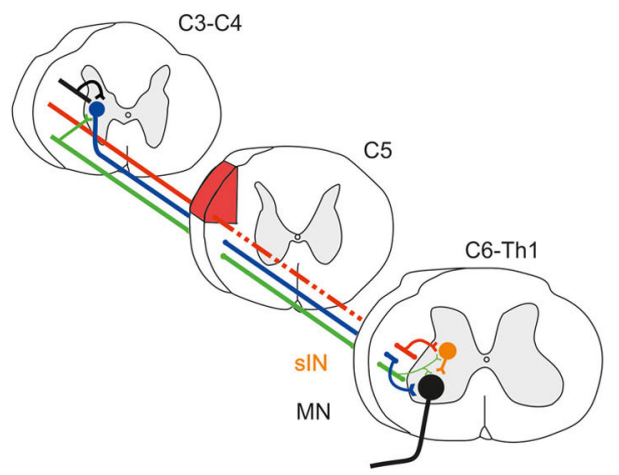

FIGURE 2 | (A) Schematic illustration of pathways remaining after a complete lesion (red area) of the DLF in C5/C6 interrupting the CST and the RuST. (B), as in (A), but for an incomplete lesion leaving a small fraction of RuST fibres intact. (C), Loss of digit grasping after the lesion in (A) (6 days postoperatively). (D) Percentage of trials with successful digit grasping in different experiments during the initial two postoperative weeks after lesion
C

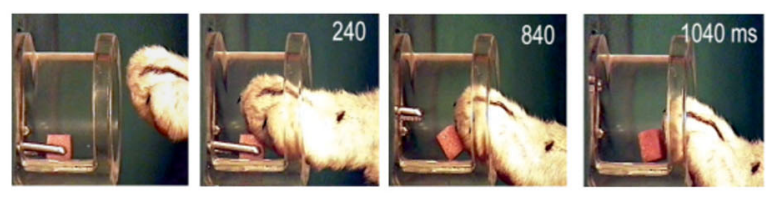

D

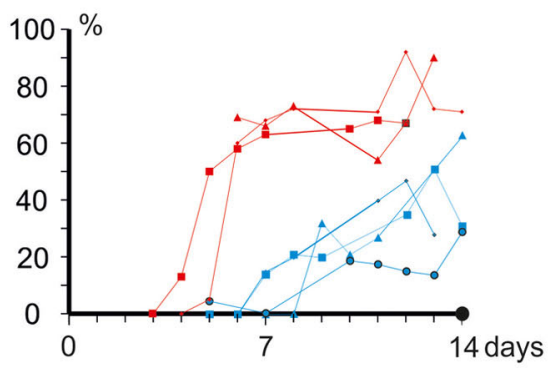

$\mathbf{E}$
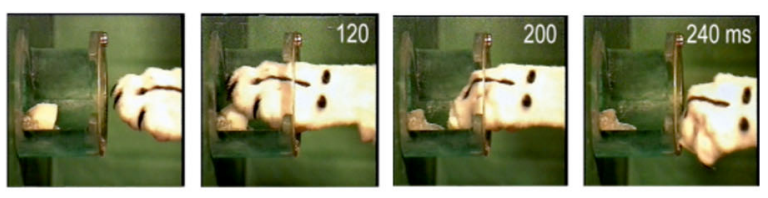

in (A) (blue plots; 4 cats) and (B) (red plots; 3 cats), respectively. The black dot indicates the absence of successful digit grasping during the first 2 weeks after lesion $(A)$ in case of less frequent training and access to morsels dropped on the floor in unsuccessful trials. (E) Successful digit grasping recorded 6 days after the lesion in (B). (C-E) Modified from Pettersson et al. (2000). takeover mediated via the ReST. This result strongly argues against that the remaining RuST fibers provided a high degree of specificity in their connections to the sINs. Rather, it suggests that the specificity was provided by the intact ReST and that the RuST fibers only gave a strengthening input to shared sINs.

A tentative explanation at network level is illustrated in Figure 4. In A is shown that the intact control of grasping exerted by the RuST is more diversified and involves more sINs than for the ReST. It is assumed that normally there are sINs with convergent inputs from the ReST and RuST. Furthermore, we assume that some of these sINs still receive convergent input after Lesion I. If so, following Lesion I, the only sINs that could mediate the descending command are those shared by the ReST and the remaining RuST. It was proposed that concomitant activity in the remaining synapses of the RuST could facilitate an induction of long term potentiation of synapses of the ReST to common sINs, in a manner of associative synaptic plasticity (Pettersson et al., 2000). During attempted digit grasping the activity of the remaining RuST synapses would then serve to direct synaptic plasticity to ReST synapses terminating on those sINs which are normally used for commanding this movement. (Pettersson et al., 2000, cf. also Pettersson et al., 2007). Since the ReST also has direct connections with the MNs, it is possible that such potentiation could be exerted also on those synapses as indicated in Figure 4B.

In order for a successful takeover, the CNS must evaluate the error in grasping following Lesion I. Such an error evaluation most likely involves the Cerebellum as shown in Figure 4C. A major source of information about spinal interneuronal activity to the cerebellum is provided by mossy fiber input via neurons in the LRN. It has been shown that both PNs in C3-C4 (Alstermark et al., 1981a) and sINs, belonging to the ipsilateral forelimb tract (Ekerot, 1990) send information to the LRN. It has been proposed that the LRN may provide the Cerebellum with an overview of linked motor behaviors like posture, reaching and grasping (Alstermark and Ekerot, 2013). If, following Lesion I, the activity in those sINs controlled by the lesioned RuST decreases, it 


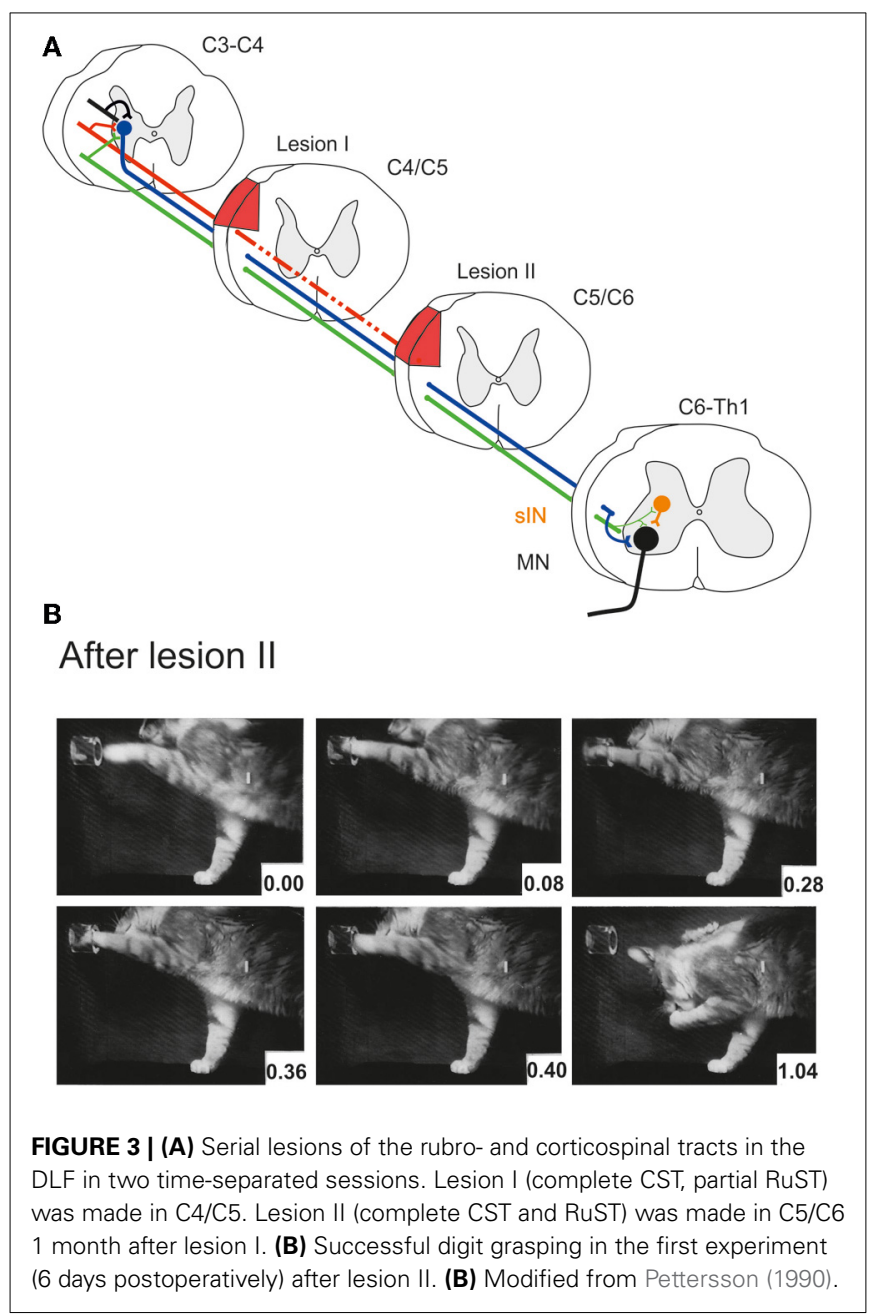

could be detected by the LRN and Cerebellum. To compensate, the Cerebellum may increase the excitation to the ReST and RuST with convergent projection to sINs. If this compensation leads to more successful grasping, this loop can be further strengthened. The function of the ascending spinal information processed by the LRN may be tested in future experiments by selective inhibition of the LRN neurons using opto-genetic techniques (Fenno et al., 2011; Miesenböck, 2011).

We have intentionally focused on the spinal mossy fibercerebellar-bulbospinal loop. There may be a control of errors via the climbing fiber-cerebellar-bulbospinal loop occurring in parallel (Ito, 1984). Also, the interaction between cerebellum-motor cortex- spinal cord is likely to play an important role (Stoodley and Schmahmann, 2010). In fact, it has been shown that there are marked excitability changes occurring in the motor cortex following spinal cord lesions (Isa and Nishimura, 2014). The augmentation of ReST output may therefore also be strengthened by increased activation of cortico-reticular pathways. An important contributor to augmented cortical modulation might be feedback control from visual pathways, as has been shown after complete spinal cord lesions in humans (Hotz-Boendermaker et al., 2011).

\section{FUNCTIONAL TAKEOVER IN PRIMATES?}

So far, it has only been possible to study a takeover in function via ReST induced by the RuST in the cat, because of the relatively dorsally located CST (Nyberg-Hansen and Brodal, 1963, 1964) that makes it possible to perform complete CST and partial RuST lesions. In the primate, the axonal locations of the CST and RuST overlap extensively, especially in the ventral part of the lateral funiculus (Poirier and Bouvier, 1966; Bortoff and Strick, 1993) and therefore it is more difficult to make selective lesions. Nevertheless, results from incomplete DLF lesions in the primate may still be of relevance. We show one example in Figure 5 which illustrates the effect of a DLF lesion on precision grip movements using the thumb and the index finger. The schematic circuitry is shown in A. Note the direct motoneuronal connections of the CST (Bernhard and Bohm, 1954), RuST (Holstege et al., 1988), ReST (Riddle et al., 2009), and the C3-C4 PN pathway (Alstermark et al., 1999). The evidence for a disynaptic corticomotoneuronal pathway via the sINs is only indirect, but suggests an effect at least to intrinsic hand muscles (Takei and Seki, 2010, 2013). Figure 5B illustrates precision grip performed the second day after a DLF lesion with incomplete transection in a macaque monkey, sparing $30 \%$ of the CST at the level of C4/C5 (Nishimura et al. unpublished data). Note the similarity in pre-and postoperative movements.

A previous report on DLF lesions with complete transection of the CST in C5 (Sasaki et al., 2004) showed successful precision grip during the first day postoperatively but with weaker grip forces so that the fingers slipped more easily and in many trials, more than one attempt were needed for successful grasping. A reduction of preshaping and increased duration of the movement was also observed (Sasaki et al., 2004). The deficits remained during an observation period of 3 months. A comparison of precision grip movements during the second postoperative day after DLF lesions with complete vs. incomplete transection of the CST is shown in the Supplementary Movie 2.

The observation of a swifter precision grip movement after partial DLF lesion in the macaque monkey resembles the findings for the (albeit less dexterous) digit grasping movement in the cat. In all likelihood the precision grip during the early postoperative period depends on the CST- and possibly also on the RuST fibers which escaped the DLF lesion. However, it remains to be investigated if the spared CST and RuST fibers can, in a longterm perspective, induce a take-over of digit grasping by other pathways such as the ReST or via the C3-C4 PN system. It is interesting that a reticulospinal pathway to arm and hand MNs has been demonstrated (Riddle et al., 2009) and that reticulospinal effects are enhanced after pyramidotomy (Zaaimi et al., 2012).

\section{USING ENDOGENOUS PLASTICITY TO ENHANCE NEURO-REHABILITATION}

The main points presented in this article are:

(1) Following a complete CST lesion and partial RuST lesion, the intact cortico-reticulospinal system, ReST, may take over the control of grasping by the help of the remaining RuST 


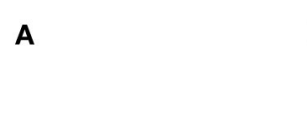

B

Intact

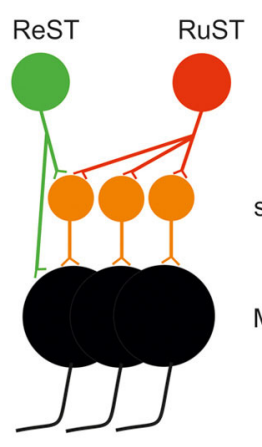

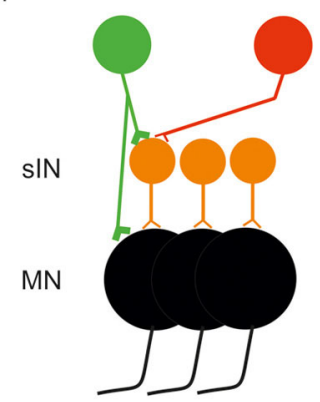

Lesion I
FIGURE 4 | Tentative explanation of facilitated takeover of digit grasping by the ReST after an incomplete DLF lesion sparing only a small fraction of the RuST (as illustrated in Figures 2B,E). (A) Before lesion, the illustrated group if sINS used for digit grasping all receive input from the RuST whereas only a minority of them from the ReST. (B) After the DLF lesion, the remaining synapses from the RuST (active during attempted digit-grasping) are assumed, by a mechanism of

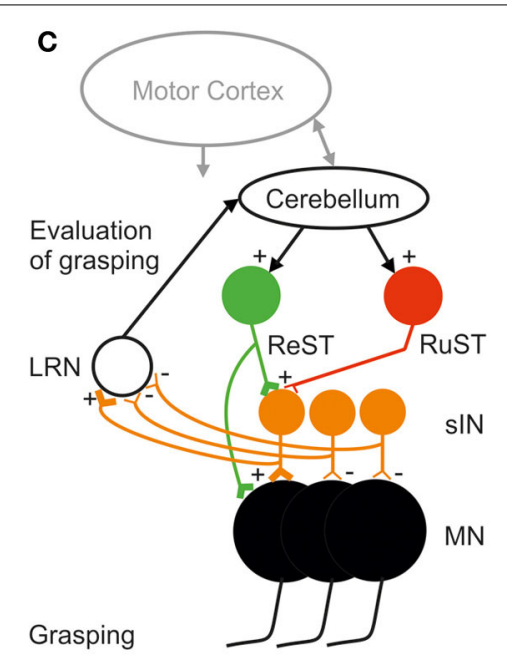

associative plasticity, to facilitate the induction of long-term potentiation in synapses from the ReST onto common sINS. (C) Mobilization of the ReST after the DLF lesion as a result of detection of reduced activity in sINS for digit grasping via ascending collaterals from them to the Lateral Reticular Nucleus (LRN). The Cerebellum may increase the activity both of ReST and RuST. The motor cortex is also important in the control of RuST and ReST (see text). fibers. This was proven in the cat, but preliminary findings in the macaque monkey suggest the possibility of a similar mechanism operating in primates.

(2) The outcome of the takeover is dependent on the remaining RuST fibers after the partial lesion. The more RuST fibers remaining, the better is the takeover via the ReST. However, a clear effect is observed even with as few as $4 \%$ remaining RuST fibers, which hypothetically could be explained by associative synaptic plasticity on common spinal INs.

(3) To evaluate the recovery, it is proposed that the Cerebellum may play an important role. It may use information from the last order premotor interneurons by virtue of their projections to the precerebellar LRN. The Cerebellum may then change the activity in the ReST and the surviving RuST neurons to compensate for the loss of control of the lesioned fibers leading to strengthening of excitatory synaptic input from sINs and ReST to MN.

These results and theoretical considerations could have a bearing on clinical rehabilitation in man and may be taken to suggest the following guide lines for the training after partial spinal cord lesions.

(1) Start the training as soon as possible after the injury. If the patient cannot perform movements, use mental imaging, visual, tactile and proprioceptive feed-back.

(2) Focus the training on skilled movements that the patient normally performs, like handling knife, fork, spoon, and chop sticks when eating, skilled typing using key-board, picking up small items with a few fingers, open/close locks using keys, buttoning a shirt and tying shoe laces.

(3) Combine training of postural control when reaching to grasp for an object.

\section{ACKNOWLEDGMENTS}

This work was supported by grants to Bror Alstermark and LarsGunnar Pettersson from the Swedish Research Council and the Human Frontier Science Program.

\section{SUPPLEMENTARY MATERIAL}

The Supplementary Material for this article can be found online at: http://www.frontiersin.org/journal/10.3389/fnins. 2014.00059/abstract

Movie 1 | Movie of two cats showing grasping of a morsel of food from a tube. Cat \#1, the first sequence was recorded preoperatively and the second sequence on the 7th postoperative day after complete transection of the CST and RuST in C5/C6. Cat \#2, the first sequence was recorded preoperatively and the second sequence on the 7th postoperative day after complete transection of the CST and partial, sparing 4\% of the RuST. The third sequence is the same as the second one, but shown at a lower frame rate.

Movie 2 | Movie of two Macaque monkeys showing grasping of a morsel of food from a tube with the thumb and index fingers. Monkey \#1, the first sequence was recorded on the 2 nd postoperative day preoperatively and the second sequence after complete transection of the CST C4/C5 (Sasaki et al., 2004). Monkey \#2, the first sequence was recorded preoperatively and the second sequence on the 7th postoperative day after partial transection of the CST sparing 30\% of these fibers (Nishimura et al. unpublished data). 


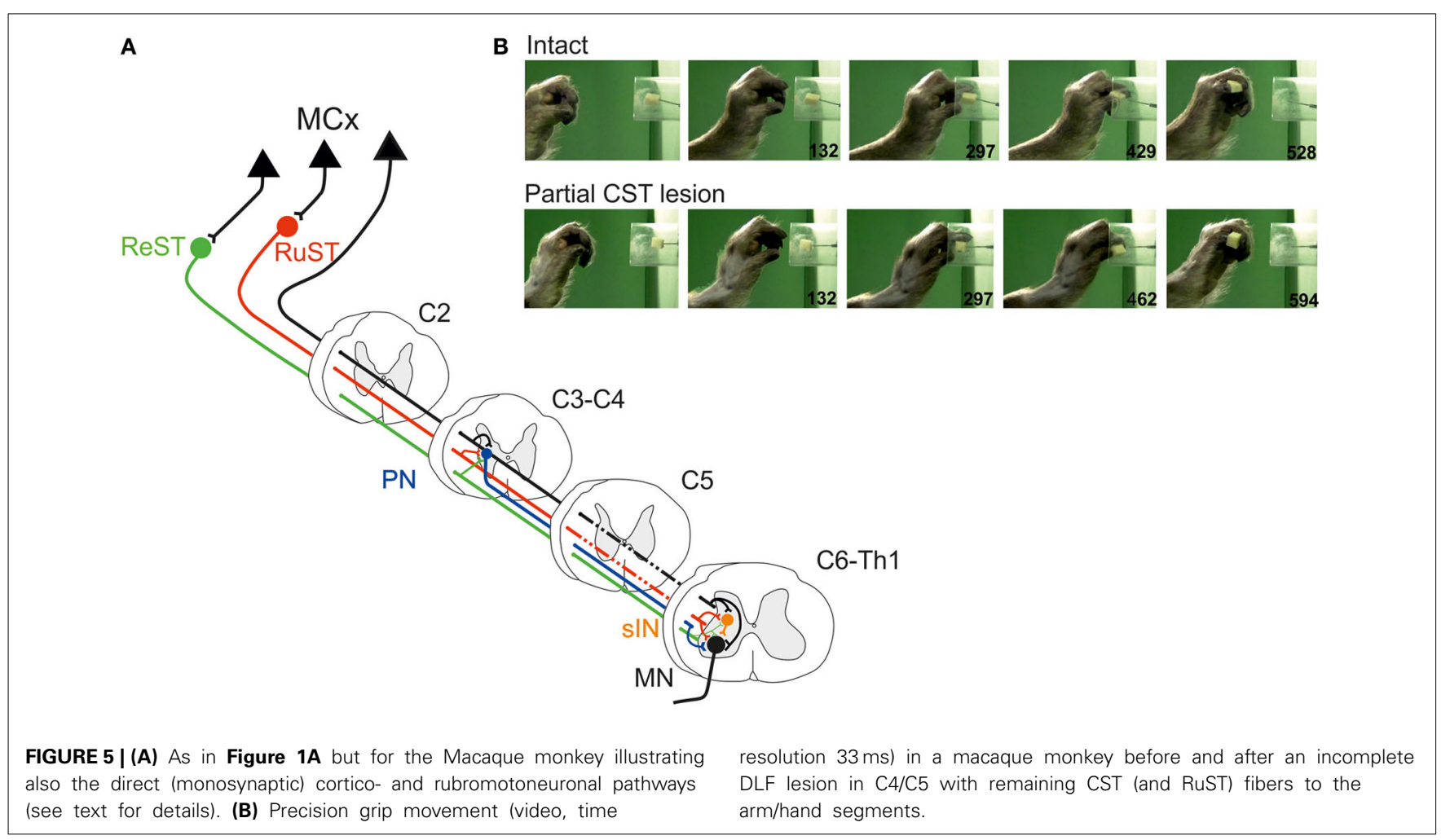

\section{REFERENCES}

Alstermark, B., and Ekerot, C. F. (2013). The lateral reticular nucleus: a precerebellar centre providing the cerebellum with overview and integration of motor functions at systems level. A new hypothesis. J. Physiol. 591, 5453-5458. doi: 10.1113/jphysiol.2013.256669

Alstermark, B., and Isa, T. (2012). Circuits for skilled reaching and grasping. Annu. Rev. Neurosci. 35, 559-578. doi: 10.1146/annurev-neuro-062111-150527

Alstermark, B., Isa, T., Ohki, Y., and Saito, Y. (1999). Disynaptic pyramidal excitation in forelimb motoneurons mediated via C3-C4 propriospinal neurons in the Macaca fuscata. J. Neurophysiol. 82, 3580-3585.

Alstermark, B., Lindström, S., Lundberg, A., and Sybirska, E. (1981a). 8. Ascending projection to the lateral reticular nucleus from $\mathrm{C} 3-\mathrm{C} 4$ propriospinal neurones also projecting to forelimb motoneurones. Exp. Brain Res. 42, 282-298. doi: 10.1007/BF00237495

Alstermark, B., and Lundberg, A. (1992). "The C3-C4 propriospinal system: targetreaching and food-taking," in Muscle Afferents and Spinal Control of Movement, eds L. Jami, E. Pierrot-Deseilligny and D. Zytnicki (Oxford: Pergamon Press), 327-354.

Alstermark, B., Lundberg, A., Norrsell, U., and Sybirska, E. (1981b). Integration in descending motor pathways controlling the forelimb in the cat. 9. Differential behavioural defects after spinal cord lesions interrupting defined pathways from higher centres to motoneurones. Exp. Brain Res. 42, 299-318. doi: 10.1007/BF00237496

Alstermark, B., Lundberg, A., Pettersson, L. G., Tantisira, B., and Walkowska, M. (1987). Motor recovery after serial spinal cord lesions of defined descending pathways in cats. Neurosci. Res. 5, 68-73. doi: 10.1016/0168-0102(87)90024-1

Alstermark, B., Pettersson, L. G., Nishimura, Y., Yoshino-Saito, K., Tsuboi, F., Takahashi, M., et al. (2011). Motor command for precision grip in the macaque monkey can be mediated by spinal interneurons. J. Neurophysiol. 106, 122-126. doi: 10.1152/jn.00089.2011

Bach-y-Rita, P. (1981). Brain plasticity as a basis of the development of rehabilitation procedures for hemiplegia. Scand. J. Rehabil. Med. 13, 73-83.

Baldissera, F., Hultborn, H. and Illert, M. (1981). "Integration in spinal neuronal systems," in Handbook of Physiology: The Nervous System, eds J. M. Brookhart, V. B. Mountcastle, and V. B. Brooks (Bethesda, MD: American Physiological Society), 509-595.
Bernhard, C. G., and Bohm, E. (1954). Cortical representation and functional significance of the corticomotoneuronal system. Arch. Neurol. Psychiatry 72, 473-502. doi: 10.1001/archneurpsyc.1954.02330040075006

Bortoff, G. A., and Strick, P. L. (1993). Corticospinal terminations in two new-world primates: further evidence that corticomotoneuronal connections provide part of the neural substrate for manual dexterity. J. Neurosci. 13, 5105-5118.

Ekerot, C.-F. (1990). The lateral reticular nucleus in the cat. VII. Excitatory and inhibitory projections from the ipsilateral forelimb tract (iF tract). Exp. Brain Res. 79, 120-128. doi: 10.1007/BF00228880

Fenno, L., Yizhar, O., and Deisseroth, K. (2011). The development and application of Optogenetics. Annu. Rev. Neurosci. 34, 389-412. doi: 10.1146/annurevneuro-061010-113817

Holstege, G., Blok, B. F., and Ralston, D. D. (1988). Anatomical evidence for red nucleus projections to motoneuronal cell groups in the spinal cord of the monkey. Neurosci. Lett. 95, 97-101. doi: 10.1016/0304-3940(88) 90639-8

Hotz-Boendermaker, S., Hepp-Reymond, M. C., Curt, A., and Kollias, S. S. (2011). Movement observation activates lower limb motor networks in chronic paraplegia. Neurorehabil. Neural Repair 25, 469-476. doi: 10.1177/1545968310389184

Isa, T., and Nishimura, Y. (2014). Plasticity for recovery after partial spinal cord injury - hierarchical organization. Neurosci. Res. 78, 3-8. doi: 10.1016/j.neures. 2013.10.008

Ito, M. (1984). The Cerebellum and Neural Control. New York, NY: Raven Press.

Miesenböck, G. (2011). Optogenetic control of cells and circuits. Annu. Rev. Cell Dev. Biol. 27, 731-758. doi: 10.1146/annurev-cellbio-100109-104051

Nyberg-Hansen, R., and Brodal, A. (1963). Sites of termination of corticospinal fibers in the cat. An experimental study with silver impregnation methods. $J$. Comp. Neurol. 120, 369-391. doi: 10.1002/cne.901200302

Nyberg-Hansen, R., and Brodal, A. (1964). Sites and mode of termination of rubrospinal fibres in the cat. An experimental study with silver impregnation methods. J. Anat. 98, 235-253.

Pettersson, L. G. (1990). Forelimb movements in the cat; kinetic features and neuronal control. Acta Physiol. Scand. Suppl. 594, 1-60.

Pettersson, L. G., Alstermark, B., Blagovechtchenski, E., Isa, T., and Sasaki, S. (2007). Skilled digit movements in feline and primate-recovery after selective 
spinal cord lesions. Acta Physiol. (Oxf). 189, 141-154. doi: 10.1111/j.17481716.2006.01650.x

Pettersson, L. G., Blagovechtchenski, E., Perfiliev, S., Krasnochokova, E., and Lundberg, A. (2000). Recovery of food-taking in cats after lesions of the corticospinal (complete) and rubrospinal (complete and incomplete) tracts. Neurosci. Res. 38, 109-112. doi: 10.1016/S0168-0102(00)00143-7

Poirier, L. J., and Bouvier, G. (1966). The red nucleus and its efferent nervous pathways in the monkey. J. Comp. Neurol. 128, 223-244. doi: 10.1002/cne.901280208

Riddle, C. N., Edgley, S. A., and Baker, S. N. (2009). Direct and indirect connections with upper limb motoneurons from the primate reticulospinal tract. J. Neurosci. 29, 4993-4999. doi: 10.1523/JNEUROSCI.3720-08.2009

Sasaki, S., Isa, T., Pettersson. L. G., Alstermark, B., Naito, K., Yoshimura, K., et al. (2004). Dexterous finger movements in primate without monosynaptic corticomotoneuronal excitation. J. Neurophysiol. 92, 3142-3147. doi: $10.1152 /$ jn. 00342.2004

Stoodley, C. J., and Schmahmann, J., D. (2010). Evidence for topgraphic organization in the cerebellum of motor control versus cognitive and affective processing. Cortex 46, 831-844. doi: 10.1016/j.cortex.2009.11.008

Sugiyama, Y., Higo, N., Yoshino-Saito, K., Murata, Y., Nishimura, Y., Oishi, T., et al. (2013). Effects of early versus late rehabilitative training on manual dexterity after corticospinal tract lesion in macaque monkeys. J. Neurophysiol. 109, 2853-2865. doi: 10.1152/jn.00814.2012

Takei, T., and Seki, K. (2010). Spinal interneurons facilitate coactivation of hand muscles during a precision grip task in monkeys. J. Neurosci. 30, 17041-17050. doi: 10.1523/JNEUROSCI.4297-10.2010
Takei, T., and Seki, K. (2013). Spinal premotor interneurons mediate dynamic and static motor commands for precision grip in monkeys. J. Neurosci. 33, 8850-8860. doi: 10.1523/JNEUROSCI.4032-12.2013

Zaaimi, B., Edgley, S. A., Soteropoulos, D. S., and Baker, S. N. (2012). Changes in descending motor pathway connectivity after corticospinal tract lesion in macaque monkey. Brain 135, 2277-2289. doi: 10.1093/brain/ aws115

Conflict of Interest Statement: The authors declare that the research was conducted in the absence of any commercial or financial relationships that could be construed as a potential conflict of interest.

Received: 20 December 2013; accepted: 14 March 2014; published online: 07 April 2014.

Citation: Alstermark B and Pettersson L-G (2014) Endogenous plasticity in neurorehabilitation following partial spinal cord lesions. Front. Neurosci. 8:59. doi: 10.3389/ fnins.2014.00059

This article was submitted to Neuroprosthetics, a section of the journal Frontiers in Neuroscience.

Copyright (๑) 2014 Alstermark and Pettersson. This is an open-access article distributed under the terms of the Creative Commons Attribution License (CC BY). The use, distribution or reproduction in other forums is permitted, provided the original author(s) or licensor are credited and that the original publication in this journal is cited, in accordance with accepted academic practice. No use, distribution or reproduction is permitted which does not comply with these terms. 